\title{
Ingestion de corps étranger : les dents de l'œesophage
}

\section{Ingestion of foreign bodies: the teeth of the esophagus}

\section{T. Joulali $\cdot$ N. Kanjaa}

Reçu le 12 janvier 2014 ; accepté le 16 février 2014

(C) SRLF et Springer-Verlag France 2014

\section{Cas clinique}

Un patient de 30 ans, sans antécédent pathologique, a été admis aux urgences pour la prise en charge d'une prothèse dentaire ingérée accidentellement lors d'un repas. Les paramètres vitaux étaient normaux avec un examen somatique et psychologique sans particularité. Après échec d'extraction instrumentale par endoscopie, le patient bénéficiait d'une thoracotomie avec extraction chirurgicale (Fig. 1). Les suites postopératoires étaient sans particularité.

\section{Commentaire}

Les corps étrangers ingérés (CE) sont nombreux, fréquents et mal répertoriés. Les enfants entre six mois et trois ans constituent plus de $80 \%$ des cas. Les personnes âgées, le plus souvent édentées représentent environ $15 \%$ des cas. La plupart des CE sont éliminés par voie naturelle. Seuls les CE dangereux par leur forme ou enclavés vont nécessiter un geste d'extraction, le plus souvent endoscopique ou chirurgical si échec d'extraction et/ou apparition de complications (perforation, infection) [1,2].

Conflit d'intérêt : T. Joulali et N. Kanjaa déclarent ne pas avoir de conflit d'intérêt.

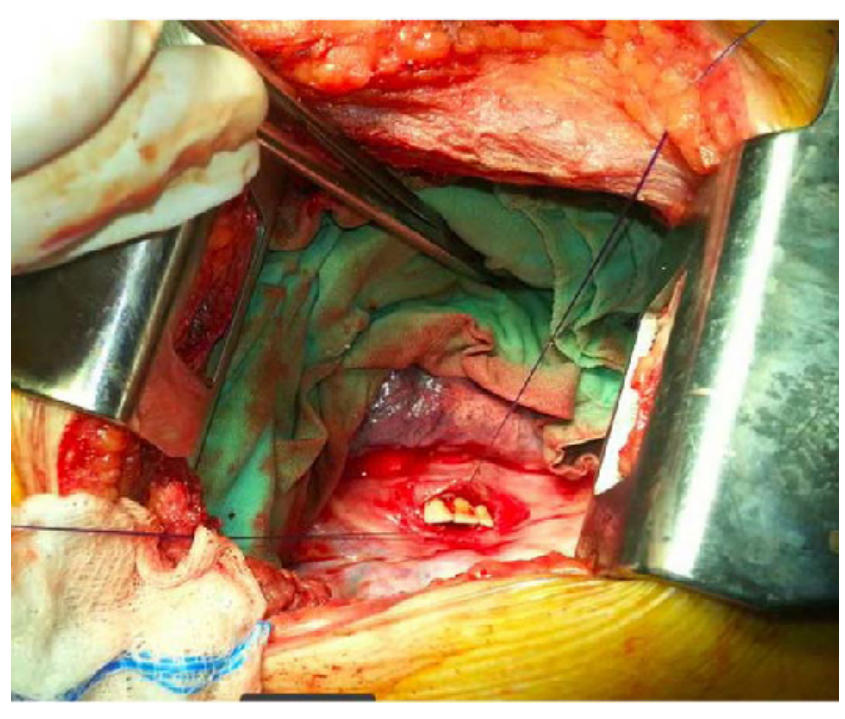

Fig. 1 Image peropératoire après ouverture de la paroi de l'œsophage montrant le corps étranger

\section{Références}

1. Hill EE, Rubel B (2008) A practical review of prevention and management of ingested/aspirated dental items. Gen Dent 56:691-4

2. Committee ASoP, Ikenberry SO, Jue TL, et al (2011) Management of ingested foreign bodies and food impactions. Gastrointest Endosc 73:1085-91 